\title{
CXCR2 and CXCR4 antagonistically regulate neutrophil trafficking from murine bone marrow
}

\author{
Kyle J. Eash, Adam M. Greenbaum, Priya K. Gopalan, and Daniel C. Link \\ Division of Oncology, Department of Medicine, Washington University School of Medicine, Saint Louis, Missouri, USA.
}

\begin{abstract}
Neutrophils are a major component of the innate immune response. Their homeostasis is maintained, in part, by the regulated release of neutrophils from the bone marrow. Constitutive expression of the chemokine CXCL12 by bone marrow stromal cells provides a key retention signal for neutrophils in the bone marrow through activation of its receptor, CXCR4. Attenuation of CXCR4 signaling leads to entry of neutrophils into the circulation through unknown mechanisms. We investigated the role of CXCR2-binding ELR ${ }^{+}$chemokines in neutrophil trafficking using mouse mixed bone marrow chimeras reconstituted with $\mathrm{Cxcr2}^{-/-}$and WT cells. In this context, neutrophils lacking CXCR2 were preferentially retained in the bone marrow, a phenotype resembling the congenital disorder myelokathexis, which is characterized by chronic neutropenia. Additionally, transient disruption of CXCR4 failed to mobilize $\mathrm{Cxcr2}^{-/-}$neutrophils. However, neutrophils lacking both CXCR2 and CXCR4 displayed constitutive mobilization, showing that CXCR4 plays a dominant role in neutrophil trafficking. With regard to CXCR2 ligands, bone marrow endothelial cells and osteoblasts constitutively expressed the ELR ${ }^{+}$chemokines CXCL1 and CXCL2, and CXCL2 expression was induced in endothelial cells during G-CSF-induced neutrophil mobilization. Collectively, these data suggest that CXCR2 signaling is a second chemokine axis that interacts antagonistically with CXCR4 to regulate neutrophil release from the bone marrow.
\end{abstract}

\section{Introduction}

Neutrophils are an essential component of the innate immune response and a major contributor to inflammation. Accordingly, the number of neutrophils in the blood is tightly regulated. Neutrophil homeostasis is maintained through a balance of neutrophil production, release from the bone marrow, and clearance from the circulation (1). The bone marrow is the primary site of neutrophil production, requiring that mature neutrophils transmigrate through an endothelial cell barrier to enter the circulation (2-4). Under basal conditions, less than $2 \%$ of the total body of mature neutrophils is in the circulation (5). Thus, the bone marrow serves as a reservoir for neutrophils that can be rapidly mobilized in response to infection or other stresses.

Major advances in our understanding of the mechanisms regulating neutrophil release from the bone marrow have come from the study of the human disease myelokathexis (with kathexis referring to retention; refs. 6-8). Myelokathexis is characterized by neutropenia despite normal to increased numbers of neutrophils in the bone marrow. It can occur in isolation or as a component of warts, hypogammaglobulinemia, infections, myelokathexis (WHIM) syndrome (WS; refs. 9, 10). Genetic studies have shown that heterozygous mutations of CXCR4 are the most common cause of WS (11). CXCR4 encodes for a CXC chemokine receptor whose major ligand is CXCL12 (also referred to as SDF-1; ref. 12). WHIM-associated mutations of CXCR4 result in the production of a carboxyterminal truncated receptor that displays impaired internalization and enhanced signaling, suggesting that excessive CXCR4 signaling may result in abnormal neutrophil retention in the bone marrow (13-16).

Conflict of interest: The authors have declared that no conflict of interest exists. Citation for this article: J Clin Invest. 2010;120(7):2423-2431. doi:10.1172/JCI41649.
Conversely, genetic deletion of CXCR4 in murine hematopoietic cells results in constitutive mobilization of neutrophils into the blood (17-22). Moreover, treatment of humans or mice with AMD3100, a small-molecule antagonist of CXCR4, causes rapid neutrophil mobilization $(23,24)$. Together with the observation that CXCL12 is constitutively expressed at a high level by bone marrow stromal cells (25), these data indicate that the CXCR4/ CXCL12 axis provides a key retention signal for neutrophils in the bone marrow.

Disruption of CXCR4 signaling is an important mechanism by which neutrophils are mobilized into the circulation under stress conditions. Treatment with G-CSF, a major mobilizing cytokine, is associated with decreased CXCL12 expression in the bone marrow and decreased CXCR4 expression on neutrophils $(5,26-28)$. Yet, the mechanisms by which attenuated CXCR4 signaling leads to migration of neutrophils toward the bone marrow venous sinuses and subsequent entry into the circulation are unclear. Based on their well-characterized role in other aspects of neutrophil biology (29), we hypothesized that ELR ${ }^{+}$CXC chemokines may direct neutrophil migration toward the bone marrow vascular space, thereby opposing the CXCR4/CXCL12 axis. This chemokine subclass, composed of CXCL1-CXCL3 and CXCL5-CXCL8, is defined by its specificity for neutrophils, signaling through CXCR1 and CXCR2, and the tripeptide motif of glutamic acid-leucine-arginine (ELR) N-terminal to the first cysteine of the CXC motif(12). ELR ${ }^{+}$chemokines are potent neutrophil chemoattractants and activators and induce neutrophil mobilization from the bone marrow when administered exogenously (4, 30-33). Accordingly, Cxcr2 ${ }^{-/-}$mice have a profound defect in neutrophil emigration to sites of inflammation, as mice lack Cxcr1 (34). With respect to neutrophil homeostasis, $C x c r 2^{-/-}$ mice housed under specific pathogen-free (SPF) conditions dis- 
A

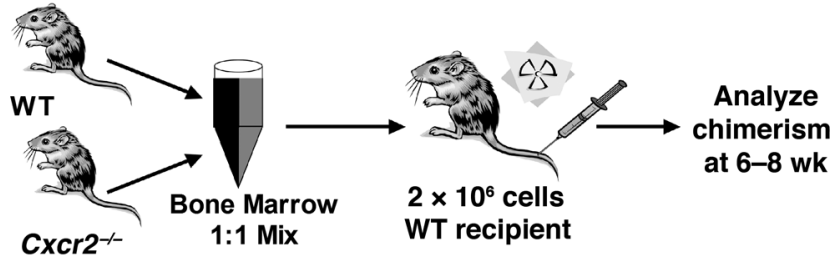

B

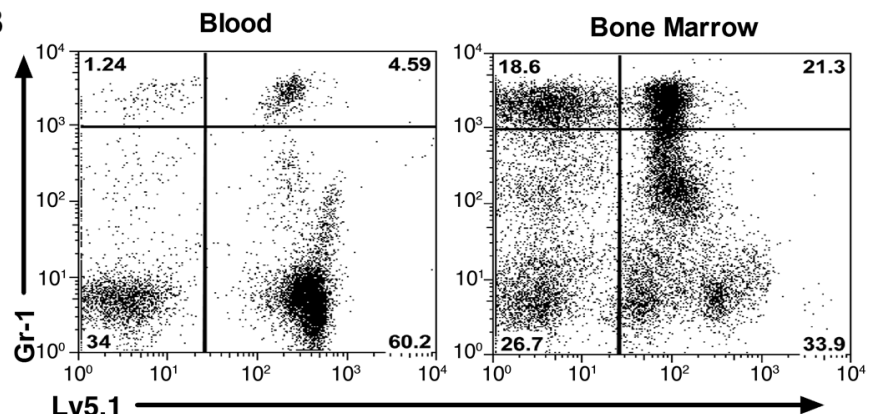

C
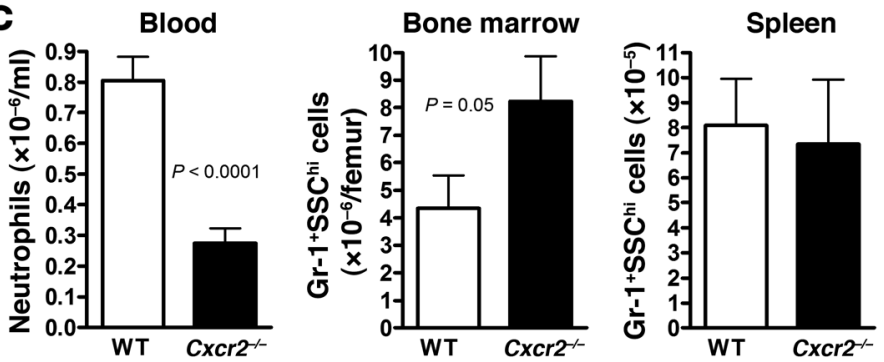

D

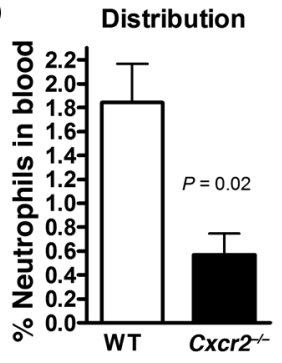

$\mathbf{E}$

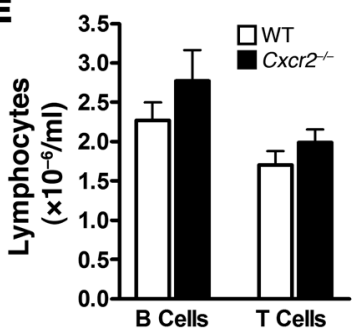

$\mathbf{F}$

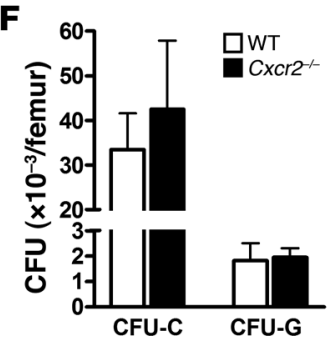

\section{Figure 1}

Cxcr2 ${ }^{-/-}$neutrophils are selectively retained in the bone marrow of mixed chimeras. (A) Generation of mixed chimeras. Bone marrow cells from WT and $\mathrm{CxCr}^{-1-}$ mice (expressing Ly5.1 and $L y 5.2$, respectively; $1 \times 10^{6}$ cells from each) were mixed in a $1: 1$ ratio and transplanted into lethally irradiated congenic WT recipients (expressing $L y 5.1$ ). Mice were analyzed 6-8 weeks after transplantation. (B) Representative dot plots showing the contribution of WT and Cxcr2- ${ }^{--}$cells (with and without $L y 5.1$, respectively) to neutrophils ( $\left.\mathrm{Gr}-1^{\text {hi }}\right)$ in the blood and bone marrow. (C) Quantitation of mature neutrophils ( $\left.\mathrm{Gr}-1^{\text {hiSSC }}{ }^{\text {hi }}\right)$ in the blood, bone marrow, and spleen. (D) NDI was calculated as described in Methods to estimate the percentage of total body neutrophils in the blood. (E) Number of B lymphocytes $\left(\mathrm{B}^{220^{+}}\right)$ or T lymphocytes (CD3+) in the blood (left) and B lymphocytes in the bone marrow (right). T lymphocyte chimerism was assessed 6 months after transplantation $(n=3)$. (F) Number of WT or $C x C r 2^{--}$CFU in culture (CFU-C) or CFU-granulocyte (CFU-G) in the bone marrow $(n=3) . n=27$ (blood); $n=6$ (bone marrow and spleen) from at least 3 independent transplantations, unless otherwise indicated. play neutrophilia with a myeloid expansion in the bone marrow and spleen. In contrast, under gnotobiotic conditions, the level of circulating neutrophils in $\mathrm{C} x \mathrm{cr} 2^{-/-}$mice is normal, raising the possibility that subclinical infections are inducing a stress granulopoiesis response (35-37).

In the present study, we generated mixed bone marrow chimeras reconstituted with WT and Cxcr2 $2^{-/}$cells to study the cellintrinsic role of CXCR2 in neutrophil trafficking. We showed that Cxcr2 $2^{-1-}$ neutrophils were selectively retained in the bone marrow, reproducing a myelokathexis phenotype. CXCR2 was required for neutrophil mobilization in response to transient CXCR 4 inhibition, although the increased release of $\mathrm{CxCr}^{-/-}$neutrophils was not altered in the absence of CXCR2 signals. CXCR2 ligands CXCL1 (also referred to as KC) and CXCL2 (also referred to as MIP-2 and GRO $\beta$ ) were constitutively expressed in bone marrow endothelium, and CXCL2 expression was induced during G-CSF-induced neutrophil mobilization. These results suggest that CXCR2 signaling is a second chemokine axis that controls neutrophil release from the bone marrow by opposing CXCR4 signals.

\section{Results}

Absence of CXCR2 results in abnormal retention of neutrophils in the bone marrow. Consistent with previous reports $(34,37)$, we observed marked neutrophilia in $\mathrm{Cxcr}^{-/-}$mice maintained under SPF conditions (absolute count, $C x \mathrm{cr}^{-/-}, 4.63 \pm 1.58 \times 10^{6}$ neutrophils $/ \mathrm{ml}$; congenic WT, $0.69 \pm 0.06 \times 10^{6}$ neutrophils $/ \mathrm{ml}$; $P=0.04)$. To determine whether this phenotype was due to a cell intrinsic effect of a loss of CXCR2 signaling, mixed bone marrow chimeras were generating by transplanting a 1:1 ratio of WT and $\mathrm{Cxcr2^{-/ }}$ bone marrow cells into irradiated congenic mice (Figure 1A). Blood neutrophil counts in the $\mathrm{Cxcr} 2^{-/-}$mixed chimeras were reduced in comparison with mice reconstituted with WT cells alone $\left(1.08 \pm 0.08 \times 10^{6}\right.$ versus $1.81 \pm 0.29 \times 10^{6}$ neutrophils $/ \mathrm{ml}$; $P=0.003$ ), which suggests that the neutrophilia in $\mathrm{Cxcr} 2^{-/-}$mice is the result of a cell-extrinsic mechanism.

In the mixed chimeras, the number of $\mathrm{Cxcr2} 2^{-/-}$neutrophils in the blood was reduced compared with that in the bone marrow (Figure 1, B and C). Whereas $65.3 \% \pm 7.6 \%$ of neutrophils in the bone marrow were derived from $\mathrm{Cxcr} 2^{-/-}$cells, only $25.0 \% \pm 3.5 \%$ 


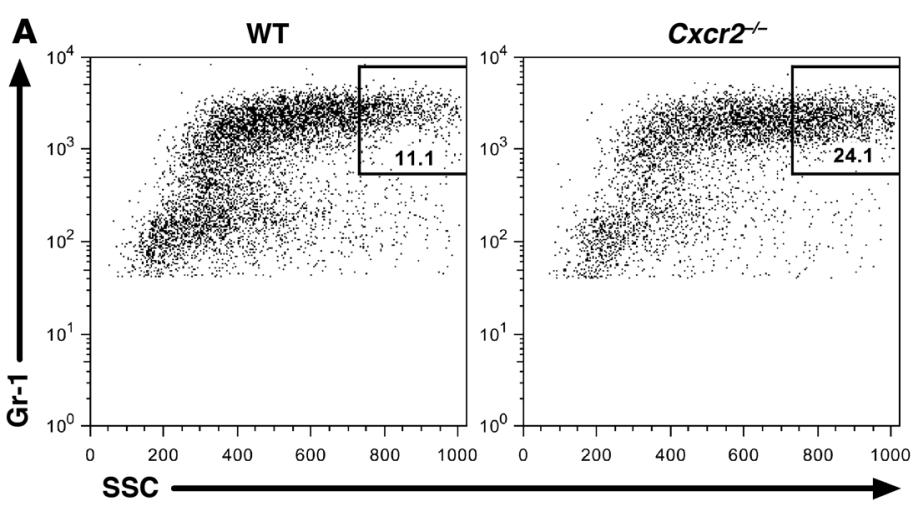

B

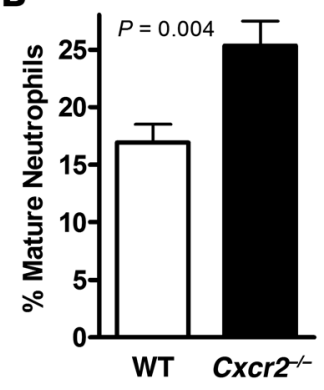

Figure 2

CXCR2 deficiency produces a myelokathexis-like phenotype. (A) Representative dot plots of mixed chimera bone marrow showing the percentage of $\mathrm{Gr}-1^{\text {hiSSChi }}$ cells within the total $\mathrm{Gr}-1^{+}$myeloid cell population for WT and $\mathrm{CxCr2^{-/- }}$ cells. (B) Percent Gr-1 ${ }^{\text {hiSSChi }}$ cells within the total $\mathrm{Gr}-1+$ myeloid cell population for $n=7$ chimeric mice from 2 independent transplants. (C) Representative photomicrographs of sorted WT and Cxcr2 ${ }^{-/-} \mathrm{Gr}-1^{+}$cells. Scale bars: $20 \mu \mathrm{m}$. (D) Manual leukocyte differentials of sorted cells from $n=5$ mice from 2 transplants. Blast, myeloblast; Band, band neutrophil; Seg, segmented neutrophil. ${ }^{\star \star \star} P<0.001,2$-way ANOVA.

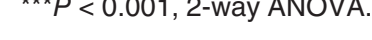

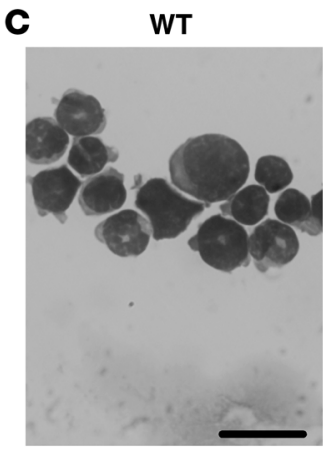
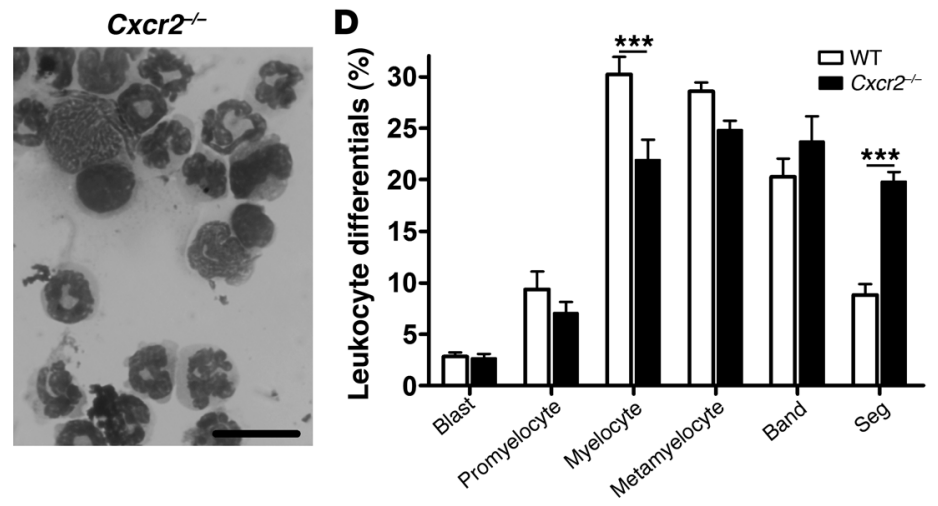

of neutrophils in the blood were from $\mathrm{Cxcr} 2^{-/-}$cells $(P<0.0001)$. Of note, the number of neutrophils in the spleen, another reservoir for neutrophils, was comparable between $\mathrm{Cxcr2^{-/- }}$ and WT cells (Figure 1C). Neutrophil trafficking from the bone marrow was estimated by calculating the percentage of neutrophils in the blood relative to the total number of neutrophils in the blood, bone marrow, and spleen (neutrophil distribution index; NDI; ref. 5). Consistent with previous studies $(22,38)$, under basal conditions, $1.84 \% \pm 0.32 \%$ of WT neutrophils were present in the blood (Figure 1D). In contrast, the percentage of $\mathrm{C} x \mathrm{Cr}^{-1-}$ neutrophils in the blood was $0.57 \% \pm 0.18 \%(P=0.02)$. No perturbation in other hematopoietic lineages was observed (Figure 1E), which indicates that the observed differences in neutrophil chimerism are not caused by altered engraftment of $\mathrm{C} x \mathrm{Cr} 2^{-/-}$hematopoietic stem cells. Consistent with this observation, the number and cytokine responsiveness of myeloid progenitors in the bone marrow were comparable between WT and Cxcr2-/- cells (Figure $1 \mathrm{~F}$ ).

Myelokathexis is characterized by the accumulation of mature, often hypersegmented or dysplastic, neutrophils in the bone marrow (10). Consistent with this phenotype, we observed that the percentage of Gr- $1^{\text {hiSSC }}{ }^{\text {hi }}$ cells - representing the most mature neutrophils (39) - relative to the total $\mathrm{Gr}-1^{+}$myeloid cell population was higher for $\mathrm{C} x \mathrm{cr} 2^{-/-}$than WT cells (Figure 2, A and B). To confirm this finding, $\mathrm{C} x \mathrm{cr} 2^{-/-}$and WT Gr-1 $1^{+}$myeloid cells were
A

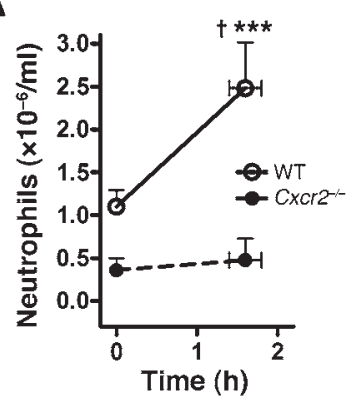

B

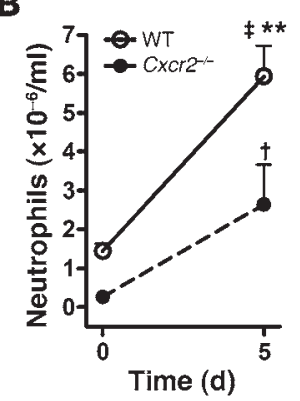

C

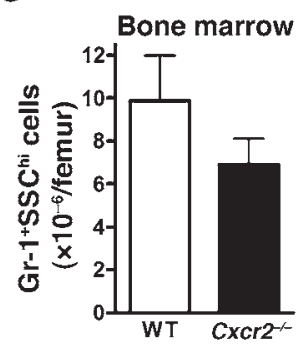

D

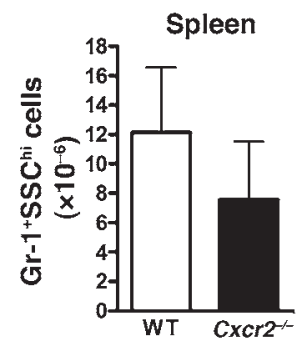

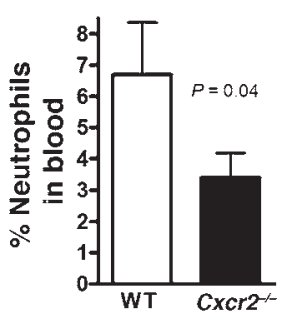

Figure 3

Mobilization of $C x c r 2^{-/-}$neutrophils by G-CSF is impaired. (A) Mixed chimeras $(n=5)$ were given a single injection of G-CSF $(125 \mu \mathrm{g} / \mathrm{kg})$, and the absolute neutrophil count for each genotype was determined 1.5 hours after injection. (B) G-CSF (125 $\mu \mathrm{g} / \mathrm{kg} / \mathrm{d}$, twice daily) was administered to a separate cohort of $n=5$ chimeric mice for 5 days, and blood neutrophils were quantified. (C) Number of WT or CxCr2 ${ }^{-/-}$Gr-1+SSChi ${ }^{+}$cells in the bone marrow and spleen after 5 days of G-CSF administration. (D) The calculated NDI after 5 days of G-CSF. $\dagger P<0.05$, $\neq P<0.01$ versus time 0 ; ${ }^{* *} P<0.01,{ }^{* *} P<0.001$ versus $C x c r 2^{-l-}$ at the same time point; 2 -way ANOVA. 
A

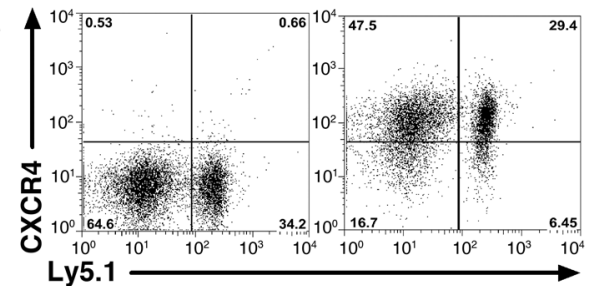

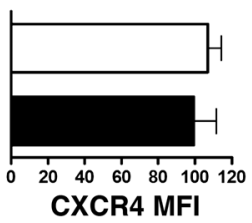

CXCR4 MFI

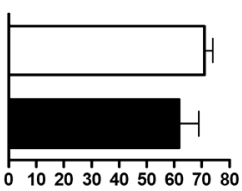

\% CXCR4 $^{+}$

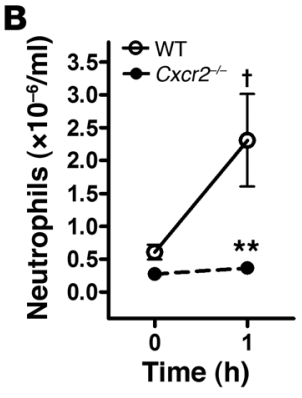

C

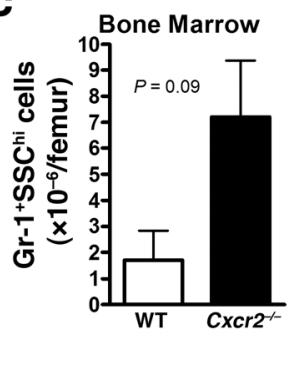

E

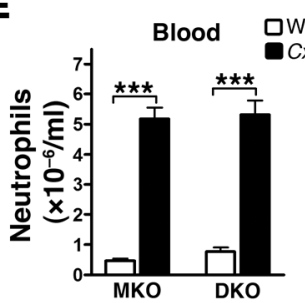

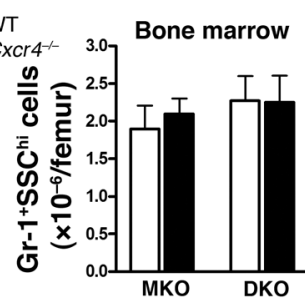
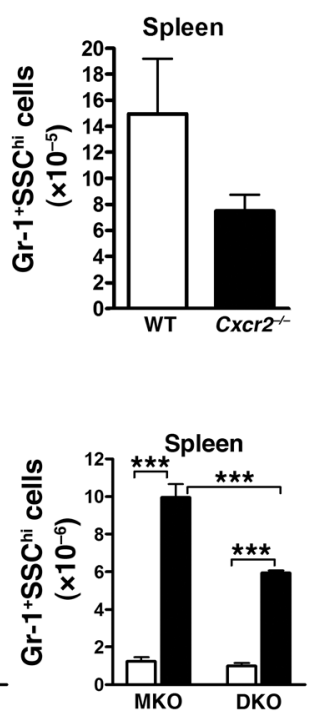

D

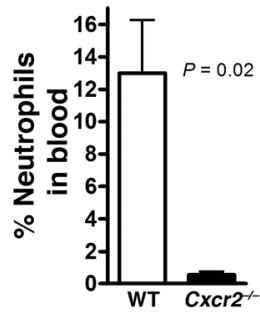

F

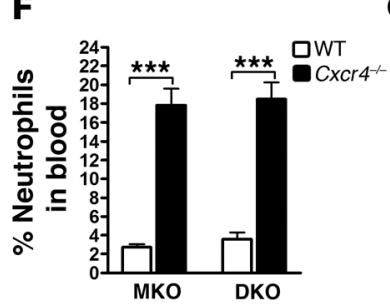

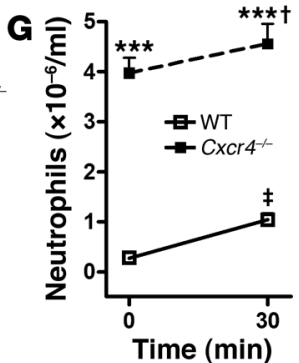

Figure 4

CXCR2 and CXCR4 signals interact antagonistically to regulate neutrophil release. (A) Representative dot plots show cell surface CXCR4 expression of WT and $\mathrm{CxCr}^{-/-} \mathrm{Gr}-1+\mathrm{SSC}$ hi bone marrow cells from a Cxcr2 ${ }^{-/-}$mixed chimera (right), and cells treated with an isotype-matched antibody (left), shown as controls. Bar graphs show CXCR4 MFI and percent CXCR4 $4^{+}$cells from $n=5$ mice. White bars, WT; black bars, Cxcr2 ${ }^{-/}$. (B) $\mathrm{Cxcr}^{-/}$mixed chimeras $(n=5)$ were given a single subcutaneous injection of AMD3100 (5 mg/kg), and neutrophils were quantified at the indicated times. (C and D) Number of neutrophils in the bone marrow and spleen (C) and NDI (D) at 1 hour after AMD3100 administration ( $n=3)$. $(\mathbf{E}$ and $\mathbf{F})$ MKO $(n=10)$ and DKO $(n=4)$ mixed chimeras were established as described in Figure 1. Blood, bone marrow, and spleen neutrophils (E) and NDI (F) were quantified 7 weeks after transplantation. ${ }^{* *} P<0.001,1$-way ANOVA. (G) MKO mixed chimeras $(n=3)$ were given a subcutaneous injection of GRO $\beta(100 \mu \mathrm{g} / \mathrm{kg})$, and the number of WT and $\mathrm{Cxcr}^{-{ }^{-}}$neutrophils in the blood was measured after 30 minutes. (B and $\mathbf{G}){ }^{\dagger} P<0.05, \neq P<0.01$ versus time $0 ;{ }^{* \star} P<0.01,{ }^{* * *} P<0.001$ versus WT at the same time point; 2 -way ANOVA.

sorted from the bone marrow of the mixed chimeras, and manual leukocyte differentials were performed. $\mathrm{Cxcr2^{-/- }}$ cells showed an increase in the proportion of highly segmented, occasionally dysplastic-appearing, neutrophils (Figure 2, C and D). Collectively, these data showed that CXCR2 deficiency results in a myelokathexis-like phenotype with a cell-intrinsic retention of neutrophils in the bone marrow.

Neutrophil mobilization by G-CSF is impaired in the absence of CXCR2. Because it is the principal cytokine regulating emergency granulopoiesis (40), we next measured the short-term (1-2 hours) and long-term (5 days) neutrophil responses to G-CSF in the Cxcr2-/mixed chimeras. Consistent with previous reports $(5,22)$, administration of G-CSF resulted in a $2.3- \pm 0.5$-fold increase in the blood of WT neutrophils within 2 hours (Figure 3A). In contrast, there was no significant increase in $\mathrm{Cxcr} 2^{-/-}$neutrophils. After the full 5 -day course of G-CSF, a significant increase in total neutrophils (both WT and $\mathrm{CxCr}^{-/-}$) in the blood, bone marrow, and spleen was observed in the mixed chimeras (Figure 3, B and C, compare with Figure $1 \mathrm{C}$ ). Note that there were fewer $\mathrm{Cxcr} 2^{-/-}$than WT neutro- phils in the bone marrow because this cohort of mice by chance had lower engraftment of $\mathrm{Cxcr} 2^{-/-}$cells (as measured by B lymphocyte chimerism; data not shown). Neutrophil release, as measured by the NDI, increased in response to 5-day G-CSF treatment in both genotypes (Figure 3D, compare with Figure 1D). However, the percentage of $\mathrm{Cxcr}^{-/-}$neutrophils in the blood after G-CSF administration was still significantly lower than that for WT cells. These data show that maximal blood neutrophil responses to G-CSF require CXCR2 signaling.

CXCR2 antagonistically regulates CXCR4-mediated neutrophil retention in the bone marrow. Previous studies have established a dominant role for CXCR4 signals in the retention of neutrophils in the bone marrow (20, 22, 41-45). Because CXCR2 signaling has previously been shown to regulate CXCR4 cell surface expression through heterologous desensitization and receptor internalization (43, 46), we first assessed CXCR4 expression on $\mathrm{CxCr} 2^{-/-}$neutrophils. However, cell surface expression of CXCR4 on bone marrow neutrophils was similar between WT and $\mathrm{Cxcr2^{-/- }}$ cells (MFI, $107 \pm 7$ and $100 \pm 12$, respectively; $P=0.4$; Figure $4 \mathrm{~A}$ ), arguing against a 
A
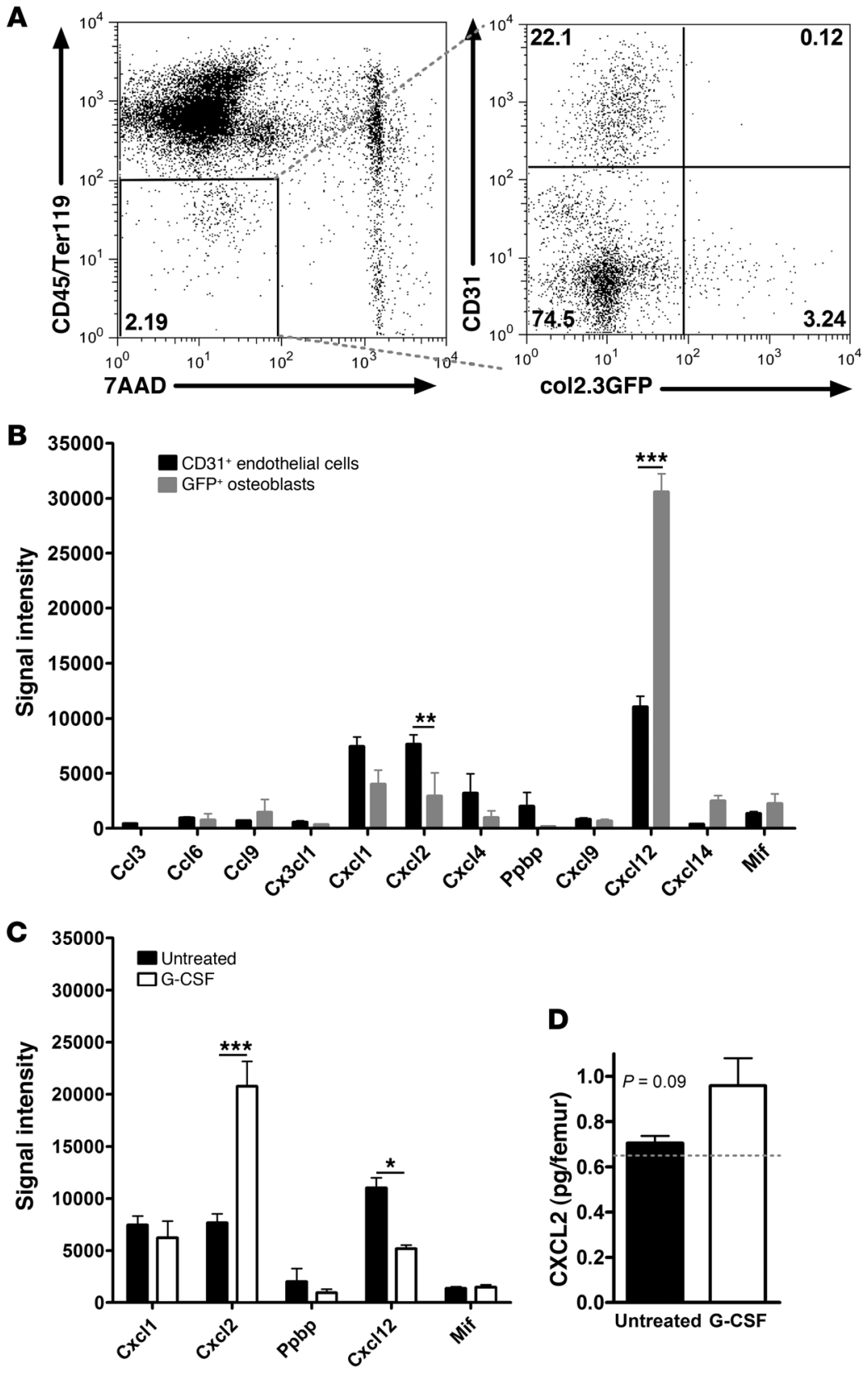

D

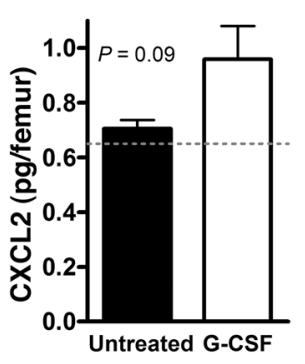

Figure 5

CXCR2 ligands are produced by bone marrow stromal cells and regulated by G-CSF. (A) Bone marrow endothelial cells $\left(7 A A D^{-} C D 45^{\circ}{ }^{\circ}\right.$ Ter $\left.119^{\circ \circ} \mathrm{CD} 31^{+}\right)$or osteoblasts (7AAD-CD45 ${ }^{\circ} \mathrm{Ter}^{119^{\mathrm{l}} \mathrm{GFP}+} \mathrm{G}^{+}$) were isolated by cell sorting from Col2.3:GFP transgenic mice. Shown are representative dot plots depicting the sorting strategy. (B) Normalized gene chip signal at baseline for all chemokines with an average signal intensity of greater than 400 in at least 1 of the cell types. When more than 1 probe set existed, the highest signal was selected. Ppbp encodes for CXCL7, and Mif is a nonchemokine ligand for CXCR2 and CXCR4 (60). (C) Expression of CXCR2 and CXCR4 ligands in endothelial cells from WT mice at baseline or after G-CSF administration. (D) CXCL2 protein in bone marrow supernatant at baseline or after G-CSF, measured by ELISA ( $n=4$ mice per group). The dashed line represents the limit of detection for the assay. ${ }^{\star} P<0.05 ;{ }^{* *} P<0.01 ;{ }^{* * *} P<0.001 ; 2$-way ANOVA.

tion in response to transient CXCR4 inhibition is dependent on CXCR2.

We previously reported that mice carrying a myeloid-specific KO of CXCR4 (LysM $\left.\mathrm{Cre}^{+} \mathrm{CXCr} 4\right)^{\mathrm{fl} / \text {; }}$ referred to herein as $\mathrm{MKO}$ ) displayed marked basal neutrophilia (22). To study the genetic interaction of the Cxcr2- and Cxcr4-null alleles, we crossed MKO mice with the Cxcr2 $2^{-/-}$mice to generate dou-

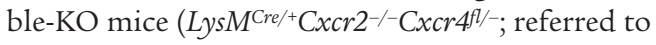
herein as DKO). Similar to the $\mathrm{C} x \mathrm{Cr}^{-/-}$and MKO mice, DKO mice displayed marked neutrophilia at baseline (data not shown). To examine the cell-intrinsic properties of neutrophils lacking both CXCR2 and CXCR4, mixed chimeras were generated as described above using DKO or, as a control, MKO bone marrow cells. Recipient mice showed the expected level of donor engraftment in the bone marrow, with $52.1 \% \pm 4.8 \%$ (DKO) and $62.2 \% \pm 2.3 \%(\mathrm{MKO})$ of B lymphocytes derived from mutant cells. As expected, mixed chimeras containing MKO cells showed a marked redistribution of $\mathrm{CxCr}^{-/-}$neutrophils into the blood (Figure 4, E and F). Surprisingly, a similar phenotype was observed in DKO chimeras, which showed that loss of CXCR2 signals cannot rescue the neu-

simple mechanism in which the absence of CXCR2 signals results in neutrophil retention through increased CXCR4 expression.

To more directly assess the relationship between CXCR2 and CXCR4 signals in the regulation of neutrophil trafficking, we treated mixed chimeras with AMD3100, a small-molecule CXCR4 antagonist. At 1 hour after AMD3100 administration, a 3.8- \pm 1.2 fold increase in WT neutrophils in the blood was observed (Figure 4B). In contrast, no increase in $\mathrm{C} x \mathrm{cr} 2^{-/-}$neutrophils in the blood was observed, despite the fact that the majority of neutrophils in the bone marrow 1 hour after AMD3100 administration were of $\mathrm{CXCR} 2^{-/-}$origin (Figure 4, B and C). Accordingly, the NDI for Cxcr2 $2^{-/}$cells after AMD3100 administration was dramatically lower than that of WT cells $(0.53 \% \pm 0.21 \%$ versus $13.0 \% \pm 3.27 \%$; $P=0.02$; Figure 4D), which suggests that neutrophil mobiliza- trophilic phenotype of CXCR4-deficient neutrophils.

To examine whether CXCR4 signals are required for mobilization by CXCR2 ligands, MKO mixed chimeras were given a single injection of the CXCR2 agonist GRO $\beta$. Whereas the number of WT neutrophils in the blood of MKO chimeras increased 3.8- \pm 0.5 -fold 30 minutes after GRO $\beta$ administration, only a minimal increase in $\mathrm{CxCr}^{-/-}$neutrophils was observed, which suggests that neutrophil release induced by CXCR2 activation is at least partially dependent on CXCR4 (Figure 4G). Collectively, these data showed that CXCR4 and CXCR2 antagonistically regulate neutrophil release from the bone marrow, with CXCR4 playing a dominant role.

Expression of chemokines by osteoblasts and endothelial cells in the bone marrow. Previous studies have established that bone marrow stromal cells, in particular osteoblasts and endothelial cells, are 
the major source of CXCL12 in the bone marrow $(21,25,27,45$, 47-50). However, the expression of other chemokines, specifically $\mathrm{ELR}^{+} \mathrm{CXCR} 2$ ligands, in bone marrow stromal cells is unknown. To address this issue, we analyzed stromal cells from the bone marrow of transgenic mice expressing GFP in osteoblast lineage cells (Col2.3-GFP; refs. 50, 51). Specifically, CD45 lo Ter119 lo stromal cells were sorted into osteoblast and endothelial fractions $\left(\mathrm{GFP}^{+}\right.$and $\mathrm{CD} 31^{+}$, respectively), which were then subjected to RNA expression profiling (Figure 5A). Of note, expression of endothelial- or osteoblast-specific genes was appropriately enriched in the relevant cell fraction, demonstrating the fidelity of our sorting strategy (Supplemental Tables 1 and 2; available online with this article; doi:10.1172/JCI41649DS1). As reported previously $(27,50)$, constitutively high expression of CXCL12 was observed in osteoblasts and endothelial cells, with higher expression in osteoblasts (Figure 5B). CXCR2 ligands CXCL1 and CXCL2 were also constitutively expressed in osteoblasts and endothelial cells, but with higher endothelial expression.

To examine the effect of G-CSF on chemokine expression in the bone marrow microenvironment, endothelial cells were isolated from the bone marrow after G-CSF administration. Osteoblasts were not sorted, since their number is markedly reduced by $\mathrm{G}-\mathrm{CSF}(27,48,50,52)$. Of note, there was no change in bone marrow endothelial cell number (D.C. Link, unpublished observation). RNA expression profiling showed that CXCL2 expression in bone marrow endothelial cells was induced $2.7- \pm 0.3$-fold by G-CSF, whereas CXCL12 mRNA was modestly reduced to $47 \% \pm 3 \%$ of its basal level; other chemokines remained unchanged (Figure 5C). Consistent with the mRNA data, CXCL2 protein was detected in the bone marrow supernatant at baseline, with increased expression after G-CSF administration, although the difference was not statistically significant (Figure 5D). Since osteoblast number is markedly reduced after G-CSF administration, these data suggest that the balance of expression in the bone marrow from proretention (CXCL12) to mobilizing chemokines (CXCL1 and CXCL2) may contribute to neutrophil mobilization by G-CSF.

\section{Discussion}

In the present study, we generated $\mathrm{Cxcr} 2^{-/-}$mixed bone marrow chimeras to characterize the cell-intrinsic effect of CXCR2 deletion on neutrophil trafficking from the bone marrow. We showed that mature, occasionally hypersegmented, $\mathrm{Cxcr} 2^{-/-}$neutrophils were selectively retained in the bone marrow, reproducing a myelokathex-

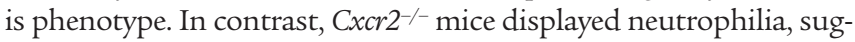
gesting augmented neutrophil production and/or release. Studies of leukocyte adhesion deficiency (LAD) mice provide a potential explanation for these discrepant results. Similar to $\mathrm{Cxcr} 2^{-/-}$mice, $\beta_{2}$ integrin-deficient mice (Itg $b 2^{--}$), which reproduce LAD type I in humans, exhibit impaired emigration of neutrophils from the circulation to peripheral tissues and neutrophilia when housed under SPF conditions $(34,53)$. In a series of elegant experiments, Forlow et al. (54) and Stark et al. (55) showed that, in Itgb2 $2^{-/-}$mixed chimeras, as little as $10 \%$ WT neutrophils was sufficient to restore normal neutrophil homeostasis. Moreover, they showed that neutrophil emigration into peripheral tissues initiated a negative feedback loop that suppresses IL-17 and G-CSF production. Thus, the emigration defect shared by $\mathrm{Cxcr2} 2^{-/-}$and Itgb2 $2^{---}$neutrophils may disrupt this negative feedback loop and lead to the production of stress cytokines that stimulate granulopoiesis. Consistent with this possibility, serum levels of IL-6 are elevated in both $\mathrm{Cxcr2} 2^{-/-}$and $\operatorname{Itg} b 2^{-/-}$mice $(34,53)$.
Our results indicate that CXCR2 and CXCR4 coordinately regulate neutrophil trafficking from the bone marrow. Previous studies have demonstrated that treatment of neutrophils with CXCR2 ligands results in impaired CXCR4 signaling through heterologous desensitization and/or internalization $(43,46)$, which suggests that CXCR2 regulates neutrophil mobilization through modulation of CXCR4 signaling and/or expression. Consistent with this possibility, Martin et al. and others previously showed that neutrophil mobilization by the CXCR2 chemokine CXCL1 was markedly increased by transient CXCR4 inhibition (42-44). Conversely, $\mathrm{Cxcr2} 2^{-/-}$neutrophils displayed almost no mobilization in response to transient CXCR4 inhibition (Figure 4, B-D). This surprising result suggests at least 2 possibilities: (a) loss of CXCR2 may augment basal CXCR4 signaling, thereby rendering cells resistant to AMD3100; and (b) CXCR2 signals may function independently of CXCR4 to direct neutrophil release after AMD3100-induced CXCR4 blockade. In support of the first possibility, doubly deficient $\mathrm{C} x \mathrm{cr}^{-/-} \mathrm{Cxcr} 4^{-/-}$neutrophils displayed constitutive mobilization (Figure 4, E and F), demonstrating that CXCR2 signals are not required for neutrophil mobilization in the complete absence of CXCR4 signals and suggesting a dominant role for CXCR4 in the regulation of neutrophil trafficking from the bone marrow. Consistent with this conclusion, the present study and our previous report showed that neutrophil mobilization by the CXCR2 chemokine GRO $\beta$ was impaired in the complete absence of CXCR4 signals (i.e., in $\mathrm{Cxcr}^{-/-}$neutrophils; Figure $4 \mathrm{G}$ and ref. 22). Collectively, these data suggest that CXCR4 and CXCR2 signaling antagonistically regulate neutrophil release from the bone marrow.

Previous studies have established that CXCL12 is constitutively expressed by bone marrow stromal cells, including osteoblasts, endothelial cells, and CXCL12-abundant reticular (CAR) cells $(21,25,27,45,47-50)$. Quantitative analysis of sorted stromal cell populations suggested that osteoblasts are the major source of CXCL12 in the bone marrow (27). Consistent with these findings, our RNA expression profiling of sorted bone marrow endothelial cells and osteoblasts demonstrated significantly higher expression of CXCL12 mRNA in osteoblasts. The expression profiling data also showed that ELR ${ }^{+}$CXC chemokines CXCL1, CXCL2, and CXCL7 were constitutively expressed in bone marrow endothelial cells and/or osteoblasts. However, in contrast to CXCL12, these chemokines were expressed at higher levels in endothelial cells. Of note, we were unable to convincingly detect CXCL1 or CXCL2 protein expression by immunostaining of bone marrow sections; thus, it is possible that other cell populations in the bone marrow may be a source of these chemokines. Together, the data suggest a tug-of-war model wherein endothelial-derived chemokines (primarily CXCR2 ligands) direct neutrophil chemotaxis toward the vasculature for entry into the circulation, while endosteal osteoblasts produce chemokines (primarily CXCL12) that promote neutrophil retention (Figure 6). Under basal conditions, the balance of chemokine production favors neutrophil retention in the bone marrow. Under stress conditions, expression of inflammatory cytokines, most notably G-CSF, is increased (56). We and others previously showed that G-CSF administration is associated with marked suppression of endosteal osteoblasts $(27,48,50,52)$. In addition, we showed here that CXCL2 expression increased in bone marrow endothelial cells after G-CSF administration. The net effect is a shift in the balance of chemokine production to the endothelium, thereby promoting neutrophil release from the bone 

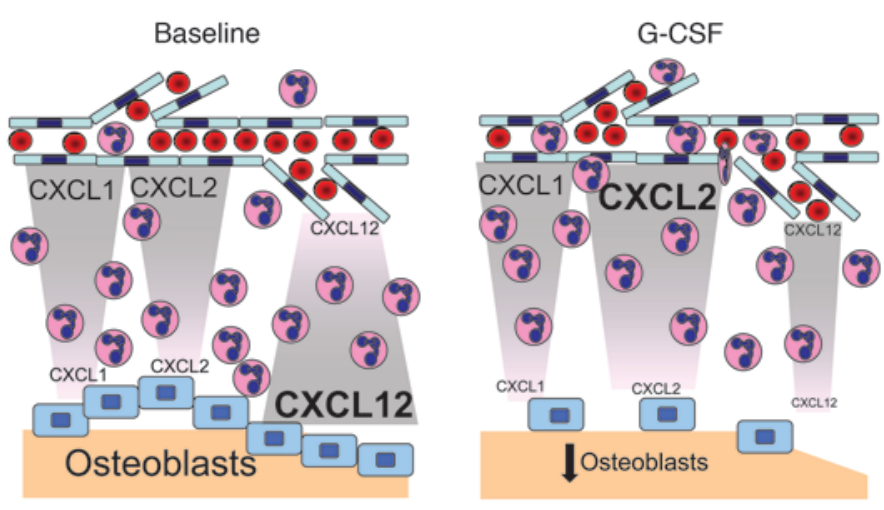

\section{Figure 6}

Tug-of-war model of neutrophil trafficking from the bone marrow. See Discussion for details.

marrow. Confirmation of this model will require demonstration that CXCL2 ligands produced by endothelial cells directly contribute to neutrophil mobilization from the bone marrow.

In summary, this study provides evidence that $\mathrm{ELR}^{+} \mathrm{CXCR} 2$ ligands are a second chemokine family that, together with CXCL12, controls neutrophil trafficking from the bone marrow. Although most cases of WS are associated with autosomal-dominant, gain-of-function mutations in $C X C R 4$, several pedigrees have been reported that lack the characteristic mutations $(9,11,14)$. Balabanian and colleagues reported a WS pedigree with decreased expression of GPCR kinase-3 (GRK3), a protein that negatively regulates CXCR4 signaling (57). Recently, homozygous loss-offunction mutations in CXCR2 have been identified in a pedigree with isolated myelokathexis (A.L. O'Shaughnessy, Q. Sun, and G.A. Diaz, unpublished observations). Our data provide experimental evidence that loss-of-function mutations in $\mathrm{Cxcr} 2$ are sufficient to induce a myelokathexis phenotype in mice. Of potential clinical relevance for the treatment of patients with myelokathexis and Cxcr2 mutations, our studies of $\mathrm{Cxcr2} 2^{-/-}$neutrophils suggest that mobilization responses to AMD3100 or G-CSF may be impaired.

\section{Methods}

Mice. Sex- and age-matched congenic C57BL/6 Cxcr2-/- (Jackson Labo-

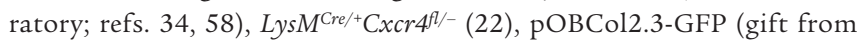
D. Rowe, University of Connecticut, Farmington, Connecticut, USA; ref. 51), and WT mice that have the Ly5.1 gene (B6.SJL-Ptprc* Pep3b BoyJ; Jackson Laboratory) were maintained under SPF conditions according to methods approved by the Washington University Animal Studies Committee. All transgenic strains had been backcrossed at least 10 generations onto a C57BL/6 background.

Bone marrow transplantation. Bone marrow cells from WT Ly5.1-expressing mice and $\mathrm{Cxcr} 2^{-/}$, MKO $\left(\mathrm{LysM}^{\mathrm{Cre} /+} \mathrm{CxCr} 4^{\mathrm{ll} /-}\right)$, or DKO $\left(\mathrm{LysM}^{\mathrm{Cre} /+} \mathrm{Cxcr2} 2^{-/-}\right.$ Cxcr $4^{7 /-}$ ) mice (Ly5.2 expressing) were mixed at a 1:1 ratio, and a total of $2 \times 10^{6}$ cells injected retro-orbitally into lethally irradiated (1,000 cGy) WT recipient mice. Antibiotics (trimethoprim-sulfamethoxazole, Alpharma) were given for 2 weeks after transplant.

Blood, bone marrow, and spleen analysis. Blood, bone marrow, and spleen cells were quantified using a Hemavet automated cell counter (CDC Technologies). Absolute neutrophil numbers were calculated assuming a blood volume of $1.8 \mathrm{ml}$ and a whole femur equivalent to $6 \%$ of the total bone marrow (59). NDI was calculated as follows: blood neutrophils/(blood neutro- phils + bone marrow neutrophils + spleen neutrophils). Bone marrow extracellular fluid was obtained by sequentially flushing both femurs with $400 \mu \mathrm{l}$ ice-cold PBS and harvesting the supernatant after pelleting cells by centrifugation at $400 \mathrm{~g}$ for 2 minutes.

Flow cytometry. Cells were stained by standard protocols with the following antibodies (eBiosciences unless otherwise noted): Chimerism was assessed using PerCP-Cy5.5-conjugated Ly5.1 (CD45.1), allophycocyanin-conjugated (APC-conjugated) Ly5.2 (CD45R.2), and 1 or more of the following lineage markers: FITC-conjugated Ly6G (Gr-1, myeloid), PE-conjugated CD3e (T lymphocytes), and APC-efluor780-conjugated CD45R (B220, B lymphocytes). A lineage cocktail of FITC-conjugated Gr-1, B220, CD3e, and Ter119 (erythroid) was used to sort progenitor cells. CXCR4 expression was assessed by incubating with anti-CD16/32 (Fc-block; BD Biosciences) and biotin-conjugated CXCR4 (BD) followed by PE-conjugated streptavidin. Isotype-matched antibodies were used as negative controls. Data were collected on a FACScan 5-color, 2-laser flow cytometer (BD Biosciences and Cytek Development) using Cellquest software (BD) and analyzed with the FlowJo software package (Tree Star).

Bone marrow lenkocyte morphology. WT or Cxcr $2^{-1-}$ bone marrow cells were isolated using a Reflection high-speed cell sorter (i-Cyt). Manual leukocyte differentials were performed in a blinded fashion on Wright-stained cytospins prepared by cytocentrifugation of 200,000 sorted cells at $18 \mathrm{~g}$ for 4 minutes. Photomicrographs were acquired using an $\mathrm{E}$ plan $\times 100 / 1.25 \mathrm{NA}$ oil immersion objective, Microphot SA microscope, Digital Sight DS-Fi1 camera, and NIS-Elements F2.30 software (all from Nikon); conversion from color to black and white was done using Photoshop CS3 (Adobe Systems).

Colony-forming cell assay. Lin ${ }^{\text {lo }}$ WT or Cxcr2 $2^{-/-}$bone marrow cells were isolated using a Reflection high-speed cell sorter, and 350 (CFU in culture) or 3,000 (CFU-granulocyte) sorted cells were plated in $2.75 \mathrm{ml}$ methylcellulose media supplemented with a cocktail of recombinant cytokines or $10 \mathrm{ng} / \mathrm{ml}$ recombinant human G-CSF (MethoCult 3434 or 3231, respectively; Stemcell Technologies). Duplicate cultures were incubated at $37^{\circ} \mathrm{C}$ for 14 days, after which colonies containing at least 100 cells were counted in a blinded fashion.

Neutrophil mobilization. Recombinant human G-CSF (Amgen) was administered by twice-daily subcutaneous injection at a dose of $125 \mu \mathrm{g} / \mathrm{kg} / \mathrm{d}$ for 5 days. Mice were analyzed 3-4 hours after the final injection on day 5. AMD3100 (Sigma-Aldrich) or recombinant human GROß (Genzyme), was administered as a single subcutaneous injection at doses of $5 \mathrm{mg} / \mathrm{kg}$ and $100 \mu \mathrm{g} / \mathrm{kg}$, respectively. Peripheral blood was obtained within 90 minutes prior to and 1-2 hours after the first injection.

Stromal cell chemokine expression profiling. Groups of $10 \mathrm{Col} 2.3-\mathrm{GFP}$ or WT mice were either left untreated or given G-CSF or vehicle for 7 days as indicated. To isolate stromal cells, femurs were serially digested with collagenase (Worthington Biochemical) as previously reported (50, 52). Stromal cells were then incubated with APC-conjugated CD45, APC-conjugated Ter119, and PE-conjugated CD31 (PECAM-1). 7-Amino-Actinomycin D (7AAD) was used to exclude nonviable cells. An average of 20,000 cells was sorted directly into TRIzoL LS reagent (Invitrogen), and RNA was isolated according to the manufacturer's instructions. RNA was amplified, processed, and hybridized to Affymetrix MOE430v2.0 GeneChip microarrays per protocols of the Siteman Cancer Center Multiplexed Gene Analysis Core Facility. After passing quality control, array data were normalized, annotated, and deposited at the Siteman Cancer Center Bioinformatics Core Facility according to standard protocols. Expression data for all known chemokines, obtained from at least 4 independent cell sorting experiments, were analyzed and compared using 2-way ANOVA.

CXCL2 ELISA. Quantification of CXCL2 protein in bone marrow extracellular fluid was performed using a commercially available ELISA kit from Peprotech according to the manufacturer's instructions. 
Statistics. Significance was determined using Prism software (GraphPad). Statistical significance of differences was calculated using 2-tailed Student's $t$ tests (assuming equal variance) or, where indicated, 1- or 2-way ANOVA with Bonferroni post-testing. $P$ values less than 0.05 were considered significant. All data are presented as mean \pm SEM.

\section{Acknowledgments}

We thank the Alvin J. Siteman Cancer Center at Washington University School of Medicine and Barnes-Jewish Hospital for the use of the High Speed Cell Sorter, Multiplexed Gene Analysis, and
Bioinformatics Cores. The Siteman Cancer Center is supported in part by NCI Cancer Center Support Grant P30 CA91842. This work was supported by NIH grant RO1 HL60772 (to D.C. Link).

Received for publication November 2, 2009, and accepted in revised form April 7, 2010.

Address correspondence to: Daniel C. Link, Division of Oncology, Department of Medicine, 660 S. Euclid Avenue, Campus Box 8007, Saint Louis, Missouri 63110, USA. Phone: 314.362.8771; Fax: 314.362.9333; E-mail: dlink@dom.wustl.edu.
1. Christopher MJ, Link DC. Regulation of neutrophil homeostasis. Curr Opin Hematol. 2007;14(1):3-8.

2. Petrides PE, Dittmann KH. How do normal and leukemic white blood cells egress from the bone marrow? Morphological facts and biochemical riddles. Blut. 1990;61(1):3-13.

3. Inoue S, Osmond DG. Basement membrane of mouse bone marrow sinusoids shows distinctive structure and proteoglycan composition: a high resolution ultrastructural study. Anat Rec. 2001; 264(3):294-304.

4. Burdon PC, Martin C, Rankin SM. Migration across the sinusoidal endothelium regulates neutrophil mobilization in response to ELR + CXC chemokines. BrJ Haematol. 2008;142(1):100-108.

5. Semerad CL, Liu F, Gregory AD, Stumpf K, Link DC. G-CSF is an essential regulator of neutrophil trafficking from the bone marrow to the blood. Immunity. 2002;17(4):413-423.

6. Zuelzer WW. "Myelokathexis"--a new form of chronic granulocytopenia. Report of a case. NEngl JMed. 1964;270:699-704.

7. Krill CEJr, Smith HD, Mauer AM. Chronic idiopathic granulocytopenia. NEngl J Med. 1964;270:973-979.

8. Bohinjec J. Myelokathexis: chronic neutropenia with hyperplastic bone marrow and hypersegmented neutrophils in two siblings. Blut. 1981;42(3):191-196.

9. Gorlin RJ, Gelb B, Diaz GA, Lofsness KG, Pittelkow MR, Fenyk JR Jr. WHIM syndrome, an autosomal dominant disorder: clinical, hematological, and molecular studies. Am J Med Genet. 2000; 91(5):368-376.

10. Diaz GA. CXCR4 mutations in WHIM syndrome: a misguided immune system? Immunol Rev. 2005; 203:235-243.

11. Hernandez PA, et al. Mutations in the chemokine receptor gene CXCR4 are associated with WHIM syndrome, a combined immunodeficiency disease. Nat Genet. 2003;34(1):70-74.

12. Murphy PM, et al. International Union of Pharmacology. XXII. Nomenclature for chemokine receptors. Pharmacol Rev. 2000;52(1):145-176.

13. Gulino AV, et al. Altered leukocyte response to CXCL12 in patients with warts hypogammaglobulinemia, infections, myelokathexis (WHIM) syndrome. Blood. 2004;104(2):444-452.

14. Balabanian K, et al. WHIM syndromes with different genetic anomalies are accounted for by impaired CXCR4 desensitization to CXCL12. Blood. 2005;105(6):2449-2457.

15. Kawai T, et al. Enhanced function with decreased internalization of carboxy-terminus truncated CXCR4 responsible for WHIM syndrome. Exp Hematol. 2005;33(4):460-468.

16. Kawai T, et al. WHIM syndrome myelokathexis reproduced in the NOD/SCID mouse xenotransplant model engrafted with healthy human stem cells transduced with C-terminus-truncated CXCR4. Blood. 2007;109(1):78-84.

17. Tachibana $\mathrm{K}$, et al. The chemokine receptor CXCR4 is essential for vascularization of the gastrointestinal tract. Nature. 1998;393(6685):591-594.

18. Zou YR, Kottmann AH, Kuroda M, Taniuchi I, Littman DR. Function of the chemokine receptor
CXCR4 in haematopoiesis and in cerebellar development. Nature. 1998;393(6685):595-599.

19. Ma Q, et al. Impaired B-lymphopoiesis, myelopoiesis, and derailed cerebellar neuron migration in CXCR4- and SDF-1-deficient mice. Proc Natl Acad Sci US A. 1998;95(16):9448-9453.

20. Ma Q Jones D, Springer TA. The chemokine receptor CXCR4 is required for the retention of B lineage and granulocytic precursors within the bone marrow microenvironment. Immunity. 1999;10(4):463-471.

21. Kawabata K, et al. A cell-autonomous requirement for CXCR4 in long-term lymphoid and myeloid reconstitution. Proc Natl Acad Sci U S A. 1999; 96(10):5663-5667.

22. Eash KJ, Means JM, White DW, Link DC. CXCR4 is a key regulator of neutrophil release from the bone marrow under basal and stress granulopoiesis conditions. Blood. 2009;113(19):4711-4719.

23. Liles WC, et al. Mobilization of hematopoietic progenitor cells in healthy volunteers by AMD3100, a CXCR4 antagonist. Blood. 2003;102(8):2728-2730.

24. Broxmeyer HE, et al. Rapid mobilization of murine and human hematopoietic stem and progenitor cells with AMD3100, a CXCR4 antagonist. J Exp Med. 2005;201(8):1307-1318.

25. Shirozu M, et al. Structure and chromosomal localization of the human stromal cell-derived factor 1 (SDF1) gene. Genomics. 1995;28(3):495-500.

26. Levesque JP, Hendy J, Takamatsu Y, Simmons PJ, Bendall LJ. Disruption of the CXCR4/CXCL12 chemotactic interaction during hematopoietic stem cell mobilization induced by GCSF or cyclophosphamide. J Clin Invest. 2003;111(2):187-196.

27. Semerad CL, et al. G-CSF potently inhibits osteoblast activity and CXCL12 mRNA expression in the bone marrow. Blood. 2005;106(9):3020-3027.

28. Kim HK, De La Luz Sierra M, Williams CK, Gulino AV, Tosato G. G-CSF down-regulation of CXCR4 expression identified as a mechanism for mobilization of myeloid cells. Blood. 2006;108(3):812-820.

29. Murphy PM. Neutrophil receptors for interleukin8 and related CXC chemokines. Semin Hematol. 1997;34(4):311-318.

30. Laterveer L, Lindley IJ, Hamilton MS, Willemze R, Fibbe WE. Interleukin-8 induces rapid mobilization of hematopoietic stem cells with radioprotective capacity and long-term myelolymphoid repopulating ability. Blood. 1995;85(8):2269-2275.

31. Opdenakker G, Fibbe WE, Van Damme J. The molecular basis of leukocytosis. Immunol Today. 1998;19(4):182-189.

32. King AG, et al. Rapid mobilization of murine hematopoietic stem cells with enhanced engraftment properties and evaluation of hematopoietic progenitor cell mobilization in rhesus monkeys by a single injection of SB-251353, a specific truncated form of the human CXC chemokine GRObeta. Blood. 2001;97(6):1534-1542.

33. Burdon PC, Martin C, Rankin SM. The CXC chemokine MIP-2 stimulates neutrophil mobilization from the rat bone marrow in a CD49d-dependent manner. Blood. 2005;105(6):2543-2548.

34. Cacalano G, et al. Neutrophil and B cell expansion in mice that lack the murine IL- 8 receptor homo- log. Science. 1994;265(5172):682-684.

35. Shuster DE, Kehrli ME Jr, Ackermann MR. Neutrophilia in mice that lack the murine IL- 8 receptor homolog. Science. 1995;269(5230):1590-1591.

36. Moore MW, Cacalano G, Wood WI, Bailish E. Response. Science. 1995;269(5230):1591.

37. Broxmeyer HE, Cooper S, Cacalano G, Hague NL, Bailish E, Moore MW. Involvement of Interleukin (IL) 8 receptor in negative regulation of myeloid progenitor cells in vivo: evidence from mice lacking the murine IL-8 receptor homologue. J Exp Med. 1996; 184(5):1825-1832.

38. Gregory AD, Hogue LA, Ferkol TW, Link DC. Regulation of systemic and local neutrophil responses by G-CSF during pulmonary Pseudomonas aeruginosa infection. Blood. 2007;109(8):3235-3243.

39. Hestdal K, et al. Characterization and regulation of RB6-8C5 antigen expression on murine bone marrow cells. J Immunol. 1991;147(1):22-28.

40. Panopoulos AD, Watowich SS. Granulocyte colony-stimulating factor: molecular mechanisms of action during steady state and 'emergency' hematopoiesis. Cytokine. 2008;42(3):277-288.

41. Nagasawa T, et al. Defects of B-cell lymphopoiesis and bone-marrow myelopoiesis in mice lacking the CXC chemokine PBSF/SDF-1. Nature. 1996; 382(6592):635-638.

42. Martin C, Burdon PC, Bridger G, Gutierrez-Ramos JC, Williams TJ, Rankin SM. Chemokines acting via CXCR2 and CXCR4 control the release of neutrophils from the bone marrow and their return following senescence. Immunity. 2003;19(4):583-593.

43. Suratt BT, et al. Role of the CXCR4/SDF-1 chemokine axis in circulating neutrophil homeostasis. Blood. 2004;104(2):565-571.

44. Wengner AM, Pitchford SC, Furze RC, Rankin SM. The coordinated action of G-CSF and ELR + CXC chemokines in neutrophil mobilization during acute inflammation. Blood. 2008;111(1):42-49.

45. Petty JM, Lenox CC, Weiss DJ, Poynter ME, Suratt BT. Crosstalk between CXCR4/stromal derived factor- 1 and VLA-4/VCAM-1 pathways regulates neutrophil retention in the bone marrow. J Immunol. 2009;182(1):604-612.

46. Richardson RM, Tokunaga K, Marjoram R, Sata T, Snyderman R. Interleukin-8-mediated heterologous receptor internalization provides resistance to HIV-1 infectivity. Role of signal strength and receptor desensitization. J Biol Chem. 2003;278(18):15867-15873.

47. Ara T, Tokoyoda K, Sugiyama T, Egawa T, Kawabata K, Nagasawa T. Long-term hematopoietic stem cells require stromal cell-derived factor- 1 for colonizing bone marrow during ontogeny. Immunity. 2003;19(2):257-267.

48. Katayama Y, et al. Signals from the sympathetic nervous system regulate hematopoietic stem cell egress from bone marrow. Cell. 2006;124(2):407-421.

49. Sugiyama T, Kohara H, Noda M, Nagasawa T. Maintenance of the hematopoietic stem cell pool by CXCL12-CXCR4 chemokine signaling in bone marrow stromal cell niches. Immunity. 2006; 25(6):977-988.

50. Christopher MJ, Liu F, Hilton MJ, Long F, Link DC. Suppression of CXCL12 production by bone mar- 
row osteoblasts is a common and critical pathway for cytokine-induced mobilization. Blood. 2009; 114(7):1331-1339.

51. Kalajzic Z, et al. Directing the expression of a green fluorescent protein transgene in differentiated osteoblasts: comparison between rat type I collagen and rat osteocalcin promoters. Bone. 2002; 31(6):654-660.

52. Christopher MJ, Link DC. Granulocyte colonystimulating factor induces osteoblast apoptosis and inhibits osteoblast differentiation. J Bone Miner Res. 2008;23(11):1765-1774.

53. Scharffetter-Kochanek K, et al. Spontaneous skin ulceration and defective $\mathrm{T}$ cell function in CD18 null mice. J Exp Med. 1998;188(1):119-131.

54. Forlow SB, Schurr JR, Kolls JK, Bagby GJ, Schwarzenberger PO, Ley K. Increased granulopoiesis through interleukin-17 and granulocyte colonystimulating factor in leukocyte adhesion moleculedeficient mice. Blood. 2001;98(12):3309-3314.

55. Stark MA, Huo Y, Burcin TL, Morris MA, Olson TS, Ley K. Phagocytosis of apoptotic neutrophils regulates granulopoiesis via IL-23 and IL-17. Immunity. 2005;22(3):285-294.

56. Kawakami M, et al. Levels of serum granulocyte colony-stimulating factor in patients with infections. Blood. 1990;76(10):1962-1964.

57. Balabanian $\mathrm{K}$, et al. Leukocyte analysis from
WHIM syndrome patients reveals a pivotal role for GRK3 in CXCR4 signaling. J Clin Invest. 2008; 118(3):1074-1084.

58. Keane MP, Belperio JA, Xue YY, Burdick MD, Strieter RM. Depletion of CXCR2 inhibits tumor growth and angiogenesis in a murine model of lung cancer. J Immunol. 2004;172(5):2853-2860.

59. Chervenick PA, Boggs DR, Marsh JC, Cartwright GE, Wintrobe MM. Quantitative studies of blood and bone marrow neutrophils in normal mice. Am J Physiol. 1968;215(2):353-360.

60. Bernhagen J, et al. MIF is a noncognate ligand of CXC chemokine receptors in inflammatory and atherogenic cell recruitment. Nat Med. 2007;13(5):587-596. 